\title{
Peridynamic Modelling of Fracture in Marine Lithium-Ion Batteries
}

\author{
Hanlin Wang, Erkan Oterkus and Selda Oterkus \\ Department of Naval Architecture, Ocean and Marine Engineering \\ University of Strathclyde \\ 100 Montrose Street \\ Glasgow G4 0LZ, United Kingdom
}

\begin{abstract}
Lithium ion battery is one of the most promising energy storage equipment due to its high energy density and long life cycle, and it has various applications in marine industry. Coupled diffusive-mechanical process during cycling has significant influence on ion diffusion and stress evolution. This may cause large deformation of electrodes which is the main reason of crack nucleation and propagation. As a consequence, this will have a negative effect on battery performance in terms of electrical capacity and thermal stability, and can cause failure of the battery. Hence, crack propagation and stress evolution during lithiation of the electrode plate is under investigation in this study. Peridynamics, as a new computational technique, is utilized for this purpose and various validation and demonstration cases were considered to demonstrate the capability of the developed framework.
\end{abstract}

Keyword: Marine lithium ion battery; Peridynamics; Failure and fracture analysis; Numerical simulation; Lithiation

\section{Introduction}

Energy conservation and emission control are the most concerning issues in marine industry. According to Marpol Annes VI regulations, the emission of nitrogen oxide in 2016 should be restricted below $80 \%$ of that in 2000 and the emission of sulfur oxide should be reduced around $90 \%$ in 2020 by comparing with the amount in 2012 [1]. As one of the solutions in emission reduction, the hybrid marine electric propulsion system has become popular in marine transportation. In this system, energy transmission and thrust operation can be directly controlled by electric power plant such as a battery. Therefore, fuel consumption and waste gas emission can be significantly reduced [2]. Marine batteries are under extensive investigation to optimise the battery performance and reduce the cost.

Based on battery applications, marine batteries can be categorised into starting batteries and deep cycle batteries [3]. Starting batteries are designed to start the marine engine with relatively high power output in a short period. Hence, starting batteries are usually composed of series of thin electrode plates to increase electrochemical reaction surface. However, a thin electrode does not have strong stiffness for harsh operating environment and does not support deep cycle charging. On the other hand, although deep-cycle batteries (or reserve batteries) may not have 
strong energy output, they have relatively large capacity which can discharge for a long time $[3,4]$. Therefore, deep-cycle marine batteries are always made to provide additional power for the auxiliary system and the propulsion system.

Based on battery chemistry, marine batteries can be classified in several types such as lead-acid batteries, lithium-ion batteries and sulphur batteries. Detailed advantages and disadvantages of different battery types are given in Table 1. Apart from cost, marine lithium ion battery is one of the most promising power storage systems due to its high energy density, low self-discharge and long life cycle [4,5]. Lithium ion batteries have various applications in marine environment such as in tugboats, icebreakers and cruise ships.

Table 1. Comparison of battery technologies [6]

\begin{tabular}{|c|c|c|}
\hline Battery type & Advantages & Disadvantages \\
\hline Lead-acid & $\begin{array}{l}\text { Low cost } \\
\text { Low self-discharge ( } 2-5 \% \text { per month) }\end{array}$ & $\begin{array}{l}\text { Short life cycle }(1200 \sim 1800 \text { cycles) } \\
\text { Life cycle affected by depth of charge } \\
\text { Low energy density (about } 40 \mathrm{Wh} / \mathrm{kg} \text { ) }\end{array}$ \\
\hline $\begin{array}{l}\text { Nickel- } \\
\text { based }\end{array}$ & $\begin{array}{l}\text { Can be fully charged (3000 cycles) } \\
\text { High energy density }(50-80 \mathrm{Wh} / \mathrm{kg})\end{array}$ & $\begin{array}{l}\text { High cost, } 10 \text { times of lead acid battery } \\
\text { High self-discharge ( } 10 \% \text { per month) }\end{array}$ \\
\hline Lithium-ion & $\begin{array}{l}\text { High energy density }(80 \sim 190 \mathrm{Wh} / \mathrm{kg}) \\
\text { Very high efficiency }(90 \sim 100 \%) \\
\text { Low self-discharge }(1 \sim 3 \% \text { per month) }\end{array}$ & $\begin{array}{l}\text { Very high cost ( } \$ 900 \sim 1300 / \mathrm{kWh}) \\
\text { Life cycle severely shorten by deep } \\
\text { discharge } \\
\text { Require special overcharge protection } \\
\text { circuit }\end{array}$ \\
\hline Sodium & High efficiency (85 92\%) & Pre-heated in stand-by mode at $325^{\circ} \mathrm{C}$ \\
\hline $\begin{array}{l}\text { Sulphur } \\
(\mathrm{NaS})\end{array}$ & $\begin{array}{l}\text { High energy density }(100 \mathrm{Wh} / \mathrm{kg}) \\
\text { No degradation of deep charge } \\
\text { No self-discharge }\end{array}$ & \\
\hline $\begin{array}{l}\text { Flow } \\
\text { battery }\end{array}$ & $\begin{array}{l}\text { Independent energy and power ratings } \\
\text { Long service life ( } 10000 \text { cycles) } \\
\text { No degradation for deep charge } \\
\text { Negligible self-discharge }\end{array}$ & Medium energy density $(40 \sim 70 \mathrm{Wh} / \mathrm{kg}$ ) \\
\hline
\end{tabular}

The performance of lithium ion battery mainly depends on the material properties of electrodes and electrolyte. The selection of electrode material is based on the operating environment of the lithium-ion batteries. For the cathode material, Cobalt compound and Nickel compound are highly electrically stable, but they are toxic and are not very thermally stable. On the other hand, manganese compound has relatively high electrical capacity and high thermal stability, but it has low cycle performance [7]. Alternatively, silicon and its compound have become attractive as battery electrode material due to its high theoretical capacity (3600 4200 $\mathrm{Ahg}^{-1}$ ), excellent cycling stability and low price $[8,15]$. However, silicon based electrodes experience large volumetric expansion (around 300\% 400\%) during lithiation [8,15,16]. Frequent cycling causes stress misdistribution which may lead to crack initiation and growth. This will have a negative influence on battery performance. Once a crack forms, stress will concentrate at the crack tips. It has been observed that large amount of lithium ions rush into the crack tip region and increase the ion concentration of this 
region. As a result, the crack tip region has relatively larger expansion as compared to other parts and the stress reduces. Hence, lithium ion diffusion and stress distribution has a direct influence on each other. To represent this interaction accurately, full coupled numerical models are necessary. So far, finite element method (FEM) has been mainly used by using cohesive zone model [8] and phase-field approach [17]. As a new numerical technique, peridynamics [9] can be a suitable alternative for FEM based models and can eliminate certain numerical issues such as convergence, mesh dependency, etc. Therefore, in this study, peridynamics has been used to predict fracture propagation during the lithiation process for the first time in the literature.

\section{Coupled lithium diffusion and stress evolution on electrode plate}

As mentioned earlier, silicon based electrodes can experience large volume expansion during the lithiation process. Frequent cycling may lead to crack initiation and growth. Once a crack emerges inside the electrode, stresses will concentrate at the crack tips. It has been observed that large amount of lithium ions quickly moves to the crack tip region and increase the ion concentration of this region. As a result, the crack tip region has relatively larger expansion by comparing with other parts and the stress reduces. This process can be expressed as [17]:

$$
\frac{\partial q}{\partial t}=M k_{B} T\left(\frac{\partial^{2} q}{\partial x^{2}}+\frac{\partial^{2} q}{\partial y^{2}}\right)-\frac{M q \Omega}{N_{A}}\left(\frac{\partial^{2} \widetilde{\sigma}}{\partial x^{2}}+\frac{\partial^{2} \widetilde{\sigma}}{\partial y^{2}}\right)-\frac{M q \Omega}{N_{A}}\left(\frac{\partial q}{\partial x} \frac{\partial \widetilde{\sigma}}{\partial x}+\frac{\partial q}{\partial y} \frac{\partial \widetilde{\sigma}}{\partial y}\right)
$$

where $q$ represents the concentration of lithium ion and $\widetilde{\sigma}$ is the hydrostatic stress which can be expressed for a two-dimensional case as [13]

$$
\sigma=\frac{1}{2}\left(\sigma_{11}+\sigma_{22}\right)
$$

with $\sigma_{11}$ and $\sigma_{22}$ being diagonal terms of the Cauchy stress tensor. The stress distribution in classical continuum mechanics can be obtained by solving the equation of motion as:

$$
\sigma_{i j, j}+b_{i}=\rho u_{i}
$$

where $b$ represents body load, $\rho$ is the mass density and $u_{i}$ is the acceleration of a material point. The stress tensor, $\sigma_{i j}$, can be expressed in terms of strains as

$$
\sigma_{i j}=\frac{E v}{(1+v)(1-2 v)} \varepsilon_{i i} \delta_{i j}+\frac{E}{(1+v)} \varepsilon_{i j}-\frac{E \Omega}{2(1-v)} q \delta_{i j}
$$

where $\varepsilon_{i j}$ is the strain tensor, $E$ and $v$ represents Elastic modulus and Poisson's ratio, respectively, $\Omega$ is the partial polar volume and $\delta_{i j}$ is the Kronecker delta. In Eq. (4) the last term represents the effect of lithium concentration on deformation field. Since 
the focus of this paper is predicting fracture, obtaining solution for Eqs. (1) and (3) is difficult since discontinuities occur as a result of crack existence and spatial derivatives at discontinuities are not defined. To analyse such cases, special treatments are necessary which may be cumbersome. Instead, these equations can be represented in a different form without using spatial derivatives, so that they are always valid regardless of derivatives. Such an approach was introduced by Silling [9] and named as peridynamics which is the numerical framework used in this study.

\section{Peridynamics}

As mentioned in Section 2, in an attempt to solve the issues due to discontinuities, peridynamics was introduced by Silling in 2000 [9]. Peridynamics is originally a continuum mechanics formulation utilizing the same continuity approximation as some other continuum mechanics formulations including Cauchy's classical continuum mechanics (CCM) formulation. Therefore, the analysis domain is assumed to be composed of infinitely small volumes called material points. In peridynamics, all material points can interact with each other within a domain of influence named Horizon, $H_{\mathbf{x}}$, as opposed to $\mathrm{CCM}$ in which the range of interactions is limited to the nearest neighbours (as shown in Fig. 1). In peridynamics, the equation of motion is also written differently with respect to CCM (see Eq. (3)) and can be expressed as

$$
\rho \ddot{\mathbf{u}}(\mathbf{x}, t)=\int_{H_{\mathbf{x}}} \mathbf{f}\left(\mathbf{u}\left(\mathbf{x}^{\prime}, t\right)-\mathbf{u}(\mathbf{x}, t), \mathbf{x}^{\prime}-\mathbf{x}\right) d V_{\mathbf{x}^{\prime}}+\mathbf{b}(\mathbf{x}, t)
$$

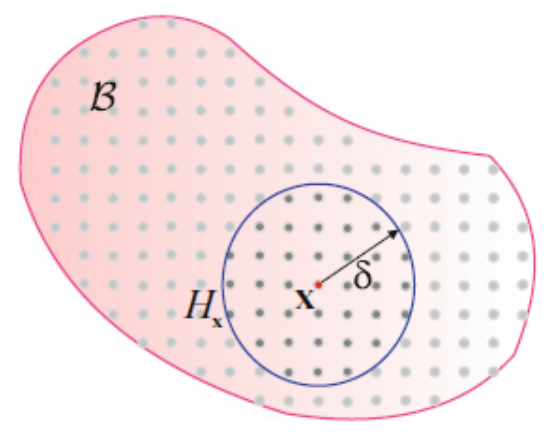

Figure 1. Horizon of the material point $\mathbf{x}[10]$

where $\mathbf{b}$ refers to body force density and $\mathbf{f}$ is the pairwise force of each bond (interaction) between material points. By defining the relative position of material points

and the relative displacement

$$
\xi=\mathbf{x}^{\prime}-\mathbf{x}
$$

$$
\eta=\mathbf{u}\left(\mathbf{x}^{\prime}, t\right)-\mathbf{u}(\mathbf{x}, t)
$$

the stretch of the bond can be calculated as: 


$$
s=\frac{|\xi+\eta|-|\xi|}{|\xi|}
$$

The bond force, $\mathbf{f}$, depends on the relative position and relative displacement of material points of a peridynamic bond. Therefore, the pairwise force can be expressed as

$$
\mathbf{f}=\frac{\xi+\eta}{|\xi+\eta|} c s
$$

In Eq. (9) $c$ is called bond constant and can be expressed in terms of material constants of CCM. For an isotropic material and a two-dimensional case, bond constant can be defined as

$$
c=\frac{9 E}{\pi h \delta^{3}}
$$

where $h$ is the thickness of the two-dimensional plate and $\delta$ is the size of the horizon. Peridynamic equation of motion can be modified by taking into account the effect of lithium diffusion as

$$
\rho \mathbf{u}=\int_{H_{\mathbf{x}}} c\left(s-\Omega q_{a v g}\right) \frac{\xi+\eta}{|\xi+\eta|} d V_{\mathbf{x}}
$$

where $q_{\text {avg }}$ refers to the average lithium ion concentration of the bond.

In peridynamics, failure definition is much simpler with respect to other techniques including well-known Linear Elastic Fracture Mechanics (LEFM). For instance, failure can be defined based on bond breakage. Once a bond stretch exceeds a critical value named as critical stretch $s_{c}$, the bond breaks permanently and the bond force between material points drops to zero. The critical stretch can be calculated in terms of the critical energy release rate of a particular material as

$$
s_{c}=\sqrt{\frac{4 \pi G_{c}}{9 E}}
$$

Once the bond breaks, the load on material point $\mathbf{x}$ will be redistributed to the remaining bonds and deformation state will change. In order to quantify the damage state of a material point, a local damage parameter of a material point $\mathbf{x}$ is defined as

$$
\varphi(\mathbf{x}, t)=1-\frac{\int_{H_{x}} \mu(\mathbf{x}, t, \xi) d V_{\mathbf{x}^{\prime}}}{\int_{H_{x}} d V_{\mathbf{x}^{\prime}}}
$$


where $\mu(\mathbf{x}, t, \xi)$ is a failure parameter and can be expressed as

$$
\mu(\mathbf{x}, t, \xi)= \begin{cases}1 & s \leq s_{c} \\ 0 & s>s_{c}\end{cases}
$$

Local damage is the percentage of broken bonds associated with the material point $\mathbf{x}$ and varies from 0 to 1 where 0 represents undamaged and 1 refers to fully damaged cases. With the growing number of broken interactions, the crack initiation and propagation can be predicted by using peridynamics. As mentioned earlier, in order to calculate the effect of deformation field on lithium concentration, it is essential to calculate the hydrostatics stress which is the summation of the diagonal terms of the Cauchy stress tensor. Cauchy stress tensor can be expressed in terms of first PiolaKirchoff stress as

where $J$ can be defined as

$$
\sigma=J \sigma_{0} F^{T}=\left[\begin{array}{ll}
\sigma_{11} & \sigma_{12} \\
\sigma_{21} & \sigma_{22}
\end{array}\right]
$$

$$
J=\operatorname{det}(F)
$$

The deformation gradient, $F$ and first Piola-Kirchoff stress, $\sigma_{0}$, can be calculated in peridynamics as [22]

$$
F=\frac{2}{\pi h \delta^{2}} \int_{H_{\mathbf{x}}} \frac{1}{|\xi|^{2}}(\xi+\eta) \otimes \xi d V_{\mathbf{x}^{\prime}}
$$

and

$$
\sigma_{0}=\int_{H_{\mathbf{x}}} \mathbf{f} \otimes\left(\mathbf{x}^{\prime}-\mathbf{x}\right) d V_{\mathbf{x}^{\prime}}
$$

Although peridynamics is originally introduced for deformation field, it is also applicable for other fields including diffusion field [23]. However, it is not straightforward to express the coupled diffusion equation in peridynamic form. In this study, this has been achieved using the peridynamic differential operator concept developed by Madenci et al. [14]. Therefore, all spatial derivatives in classical diffusion equation given in Eq. (1) can be transformed into peridynamic form as

$$
\begin{aligned}
& \frac{\partial f(\mathbf{x}, t)}{\partial x}=\left(\frac{2}{\pi h \delta^{2}}\right) \int_{H_{\mathbf{x}}}\left(\frac{f\left(\mathbf{x}^{\prime}, t\right)-f(\mathbf{x}, t)}{|\xi|}\right) \cos \theta d V_{\mathbf{x}^{\prime}} \\
& \frac{\partial f(\mathbf{x}, t)}{\partial y}=\left(\frac{2}{\pi h \delta^{2}}\right) \int_{H_{\mathbf{x}}}\left(\frac{f\left(\mathbf{x}^{\prime}, t\right)-f(\mathbf{x}, t)}{|\xi|}\right) \sin \theta d V_{\mathbf{x}^{\prime}}
\end{aligned}
$$




$$
\begin{aligned}
& \frac{\partial^{2} f(\mathbf{x}, t)}{\partial x^{2}}=\left(\frac{3}{\pi h \delta^{3}}\right) \int_{H_{\mathbf{x}}}\left(\frac{f\left(\mathbf{x}^{\prime}, t\right)-f(\mathbf{x}, t)}{|\xi|}\right)\left(3 \cos ^{2} \theta-\sin ^{2} \theta\right) d V_{\mathbf{x}^{\prime}} \\
& \frac{\partial^{2} f(\mathbf{x}, t)}{\partial y^{2}}=\left(\frac{3}{\pi h \delta^{3}}\right) \int_{H_{\mathbf{x}}}\left(\frac{f\left(\mathbf{x}^{\prime}, t\right)-f(\mathbf{x}, t)}{|\xi|}\right)\left(3 \sin ^{2} \theta-\cos ^{2} \theta\right) d V_{\mathbf{x}^{\prime}}
\end{aligned}
$$

with $f=q$ and $\tilde{\sigma}$, and $\theta$ is the orientation of the bond with respect to $x$-axis. Substituting expressions given in Eqs. (19a,d) in Eq. (1) yields peridynamic diffusion equation as

$$
\begin{aligned}
\frac{\partial q}{\partial t} & =\left(\frac{6 M k_{B} T}{\pi h \delta^{3}}\right) \int_{H_{\mathbf{x}}}\left(\frac{q\left(\mathbf{x}^{\prime}, t\right)-q(\mathbf{x}, t)}{|\xi|}\right) d V_{\mathbf{x}^{\prime}}-\left(\frac{6 M q \Omega}{\pi h \delta^{3} N_{A}}\right) \int_{H_{\mathbf{x}}}\left(\frac{\tilde{\sigma}\left(\mathbf{x}^{\prime}, t\right)-\tilde{\sigma}(\mathbf{x}, t)}{|\xi|}\right) d V_{\mathbf{x}^{\prime}} \\
& -\left(\frac{4 M q \Omega}{\pi^{2} h^{2} \delta^{4} N_{A}}\right)\left(\int_{H_{\mathbf{x}}}\left(\frac{q\left(\mathbf{x}^{\prime}, t\right)-q(\mathbf{x}, t)}{|\xi|}\right) \cos \theta d V_{\mathbf{x}^{\prime}}\right)\left(\int_{H_{\mathbf{x}}}\left(\frac{\tilde{\sigma}\left(\mathbf{x}^{\prime}, t\right)-\tilde{\sigma}(\mathbf{x}, t)}{|\xi|}\right) \cos \theta d V_{\mathbf{x}^{\prime}}\right) \\
& -\left(\frac{4 M q \Omega}{\pi^{2} h^{2} \delta^{4} N_{A}}\right)\left(\int_{H_{\mathbf{x}}}\left(\frac{q\left(\mathbf{x}^{\prime}, t\right)-q(\mathbf{x}, t)}{|\xi|}\right) \sin \theta d V_{\mathbf{x}^{\prime}}\right)\left(\int_{H_{\mathbf{x}}}\left(\frac{\tilde{\sigma}\left(\mathbf{x}^{\prime}, t\right)-\tilde{\sigma}(\mathbf{x}, t)}{|\xi|}\right) \sin \theta d V_{\mathbf{x}^{\prime}}\right)
\end{aligned}
$$

The details of the derivation of Eq. (20) and its validation by comparing against finite element method results are presented in Wang et al. [24].

\section{Numerical Results}

In order to validate the developed formulation and demonstrate its capabilities, a squared-shape silicon specimen with a pre-existing crack is considered as shown in Fig. 2 which was previously analysed by Zuo and Zhao [17] using phase-field approach. The thickness of the specimen plate is very small compared with its length and width. Therefore, the plane stress assumption is utilized in order to simplify the numerical calculations. External boundaries are free from displacement constraints. The plate is subjected to maximum lithium-ion concentration, $q_{\max }$, on all of its boundaries by introducing a fictitious region with a thickness of horizon size along its boundaries [25]. As the time progresses, lithium ions start to diffuse from external boundaries into the internal part of the plate. 


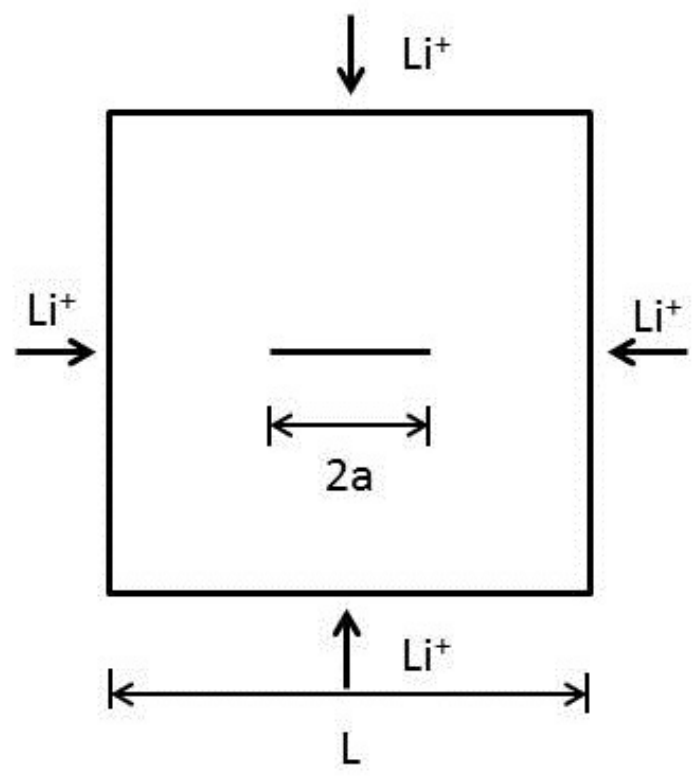

Figure 2: Pre-cracked square shape electrode plate specimen [17]

Parameters used in the peridynamic analysis are given in Table 2.

Table 2: Parameters in numerical simulation

\begin{tabular}{ccc}
\hline$E$ & Elastic constant of silicon & $80 \mathrm{GPa}$ \\
$v$ & Poisson's ratio of silicon & 0.22 \\
$\Omega$ & partial molar volume & $8.5 \times 10^{-6} \mathrm{~m}^{3} \mathrm{~mol}^{-1}$ \\
$M$ & molecular mobility & $500 \mathrm{~m}^{2} \mathrm{~J}^{-1} \mathrm{~s}^{-1}$ \\
$k_{B}$ & Boltzmann constant & $1.38 \times 10^{-23} \mathrm{~J} \cdot \mathrm{K}^{-1}$ \\
$T$ & absolute temperature & $300 \mathrm{~K}$ \\
$N_{A}$ & Avogadro's constant & $6.02 \times 10^{23} \mathrm{~mol}^{-1}$ \\
$\varepsilon_{c}$ & threshold strain & 0.04 \\
$q_{\max }$ & maximum concentration & $1.18 \times 10^{4} \mathrm{molm}^{-3}$ \\
\hline
\end{tabular}

As mentioned earlier, in peridynamics, failure takes place if the stretch of a bond exceeds a critical value according to Eq. (14). The critical stretch value for the electrode material is specified as 0.04 .

\subsection{Coupled analysis for a single crack}

In the first case, the width of the square plate is 1 micrometer. A stationary crack with a length of $20 \%$ of the length of the specimen plate lies horizontally in the central region. The initial damage of the plate can be obtained by breaking all peridynamic bonds passing through the crack surface (Fig. 3a). As the lithiation progresses, the boundary region of the plate expands first (Fig. $3 b$ and 3c). As a result of this deformation, the boundary region is subjected to a compressive stress state while the 
central region is subjected to a tensile stress state. Since the crack is located at the central part of the plate, the stress concentrates at the two crack tips and the maximum tensile stress becomes approximately 420MPa (Fig. 3d). According to Eq. 1, the high pressure gradient at crack tip regions is the main motivation of lithium ion enrichment and "two dots" on Fig. 3e represent higher lithium ion concentration at the crack tip area.
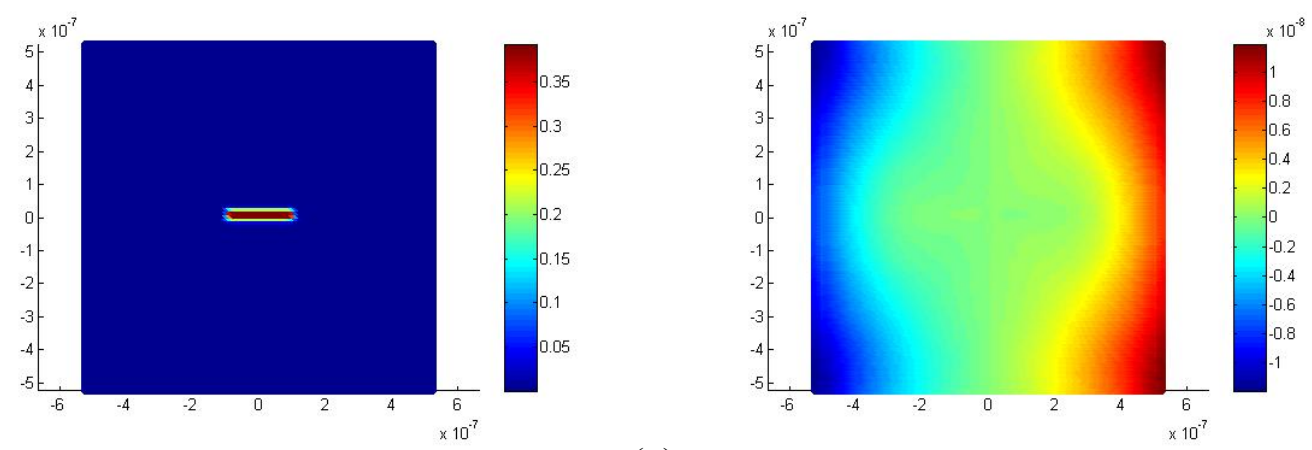

(a)

(b)
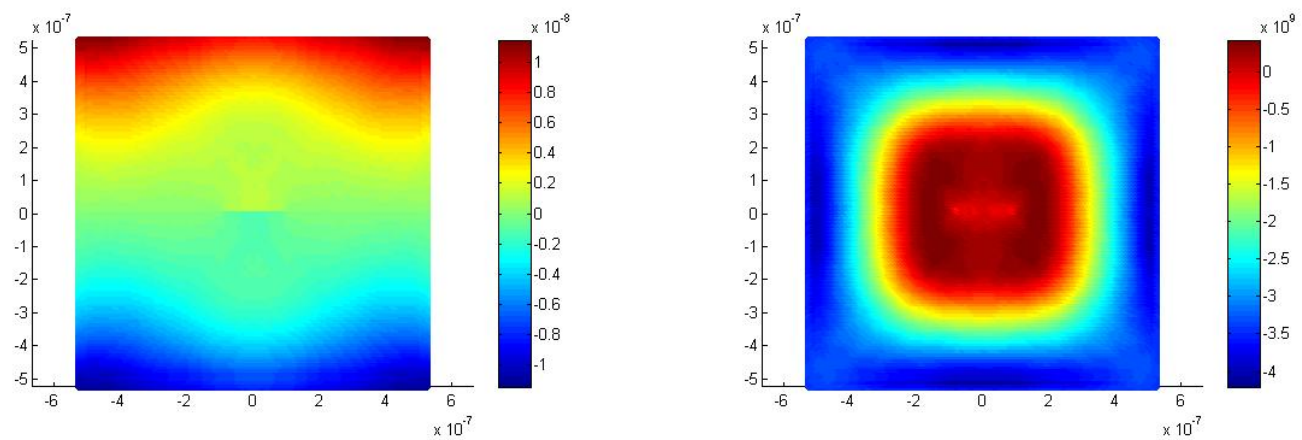

(c)

(d)

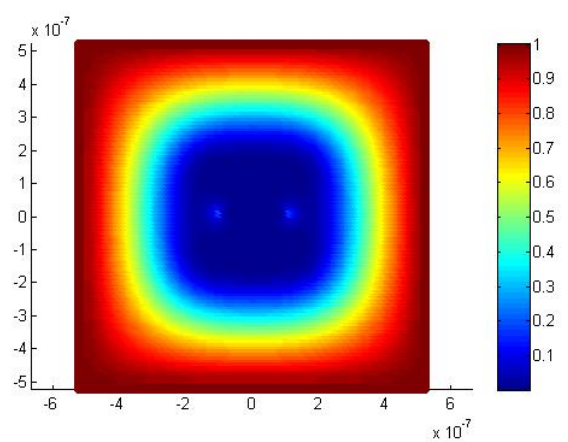

(e)

Figure 3: Results of the electrode plate with a stationary crack: (a) Initial damage (b) Displacement in $x$ direction (c) Displacement in y direction (d) Hydrostatic stress (e) Lithium ion concentration

The electrode material in this simulation is not strong enough to withstand high hydrostatic stress (i.e. 420MPa). Therefore, crack propagates to decrease strain energy release rate and increase surface energy of the crack as shown in Figs. 4a-f. Note that, 
the high lithium concentration region at the crack tips moves as crack propagates (Fig. $4 f)$.
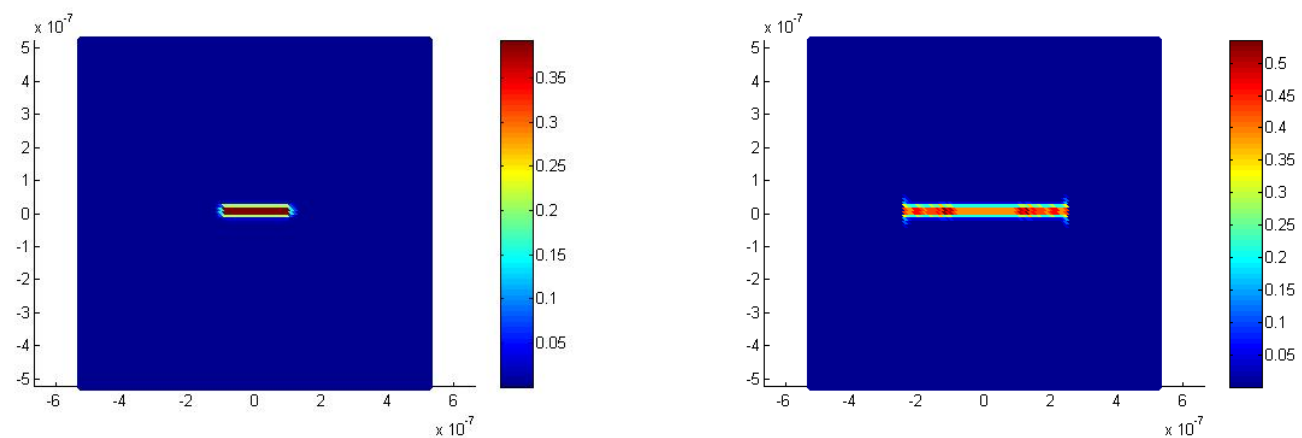

(a)

(b)
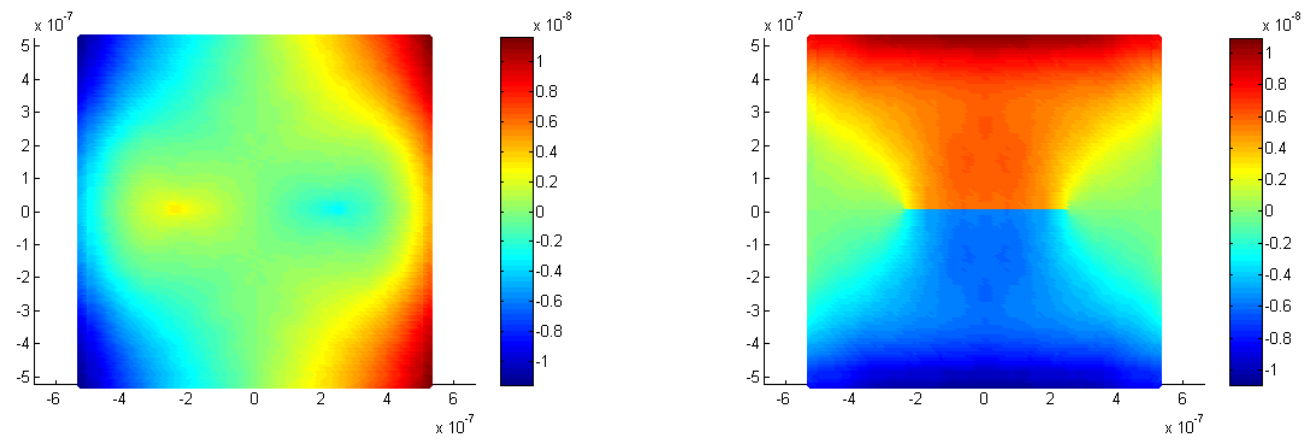

(c)

(d)
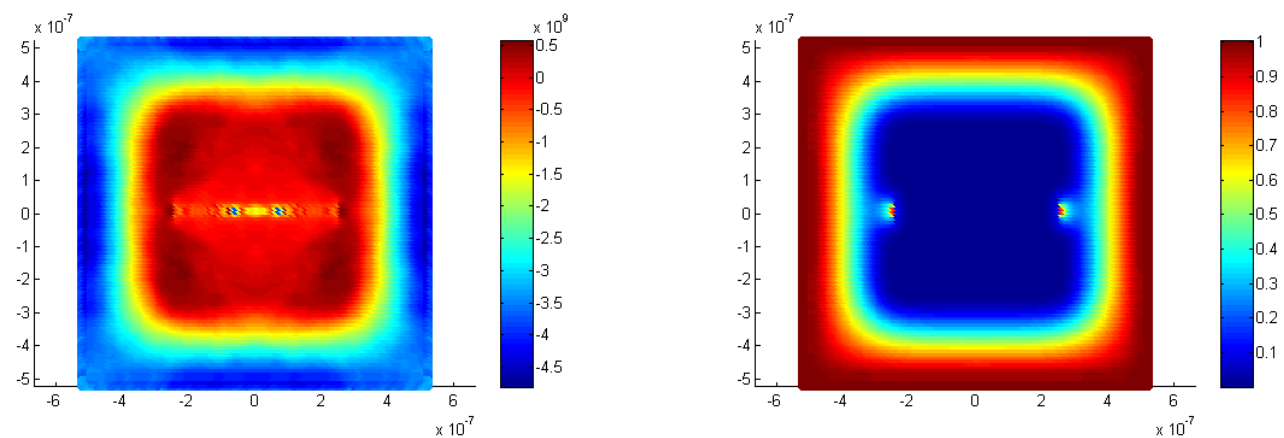

(e)

Figure 4: Results of the electrode plate after allowing crack growth: (a) Initial damage (b) Damage after deformation (c) Displacement in $\mathrm{x}$ direction (d) Displacement in y direction (e) Hydrostatic stress (f) Lithium ion concentration

\subsection{Coupled analysis for multiple cracks}

Electrodes may have multiple initial damages due to manufacturing quality, transportation and some unexpected circumstances. Therefore, a single crack may not be sufficient to describe the damage state of a battery electrode. Hence, in this case study, an electrode plate with two cracks with different orientations is investigated.

Similar to Section 4.1, an electrode plate specimen with 1 micrometer in size is considered. In the first case, a pair of cracks parallel to each other lies horizontally at 
the center of the plate (shown as Fig. 5a). This pair of cracks has the same length which is $10 \%$ of the plate width. Distance between the pair cracks is $5 \%$ of the plate width. As lithium ions diffuse from boundaries to central region, the lithium ion concentration at crack tip regions increases (shown as Fig.5d). However, multiple parallel cracks cause decrease in strain energy release rate and increase in crack surface energy of the electrode plate. Hence, the propagation of these cracks is not that obvious as in Section 4.1. These cracks have a potential to combine into a larger crack as shown in Fig. 5b. However, a newly formed crack perpendicular to the horizontal cracks emerges with a trend connecting the initial cracks into a single crack. This new crack cannot propagate further vertically since it is under "protection" of the initial cracks. [19,20]

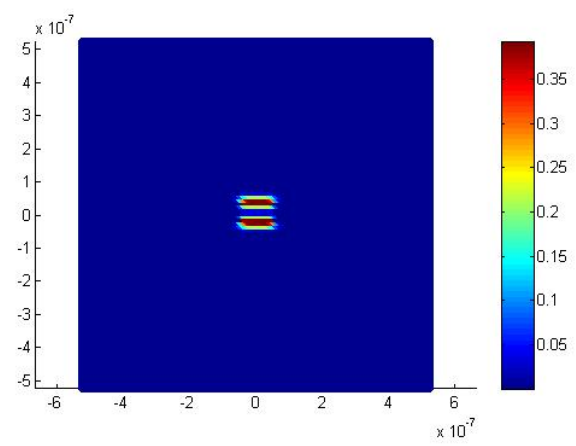

(a)

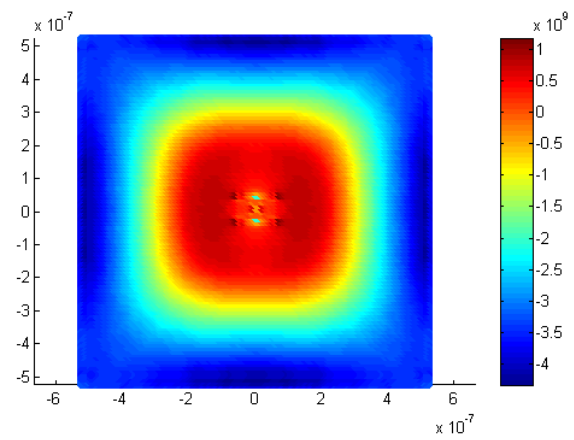

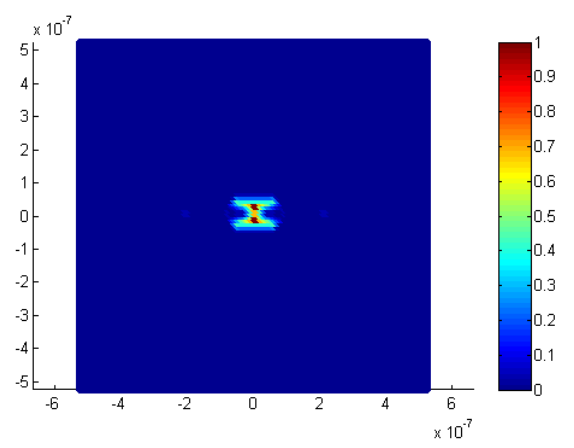

(b)

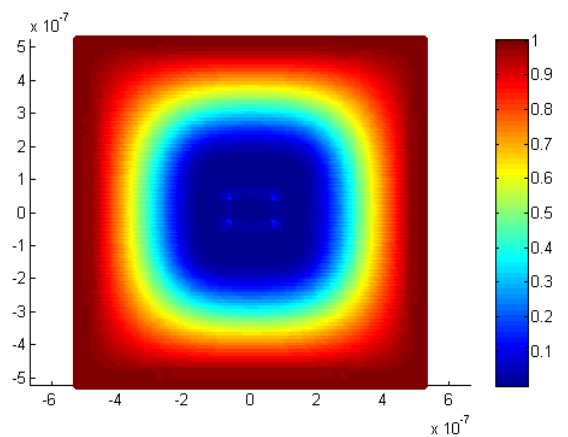

(d)

Figure 5: Results of the electrode plate with parallel cracks: (a) Initial damage (b) Damage after deformation (c) Hydrostatic stress (d) Lithium ion concentration

Cracks can also align initially perpendicular to each other as shown in Fig. 6a. The size of the horizontal crack is $12 \%$ of the plate length and the size of vertical crack is $10 \%$ of the plate length. The distance between these cracks is $10 \%$ of the plate length. As lithium ion diffuses, the vertical crack grows first and merges with horizontal crack, forming a " $T$ " shape crack. Then the horizontal crack starts to propagate upwards on two crack tips as shown in Fig. 6b. High pressure values at the crack tips also contribute to crack merging as shown in Figs. 6c and 6d. 

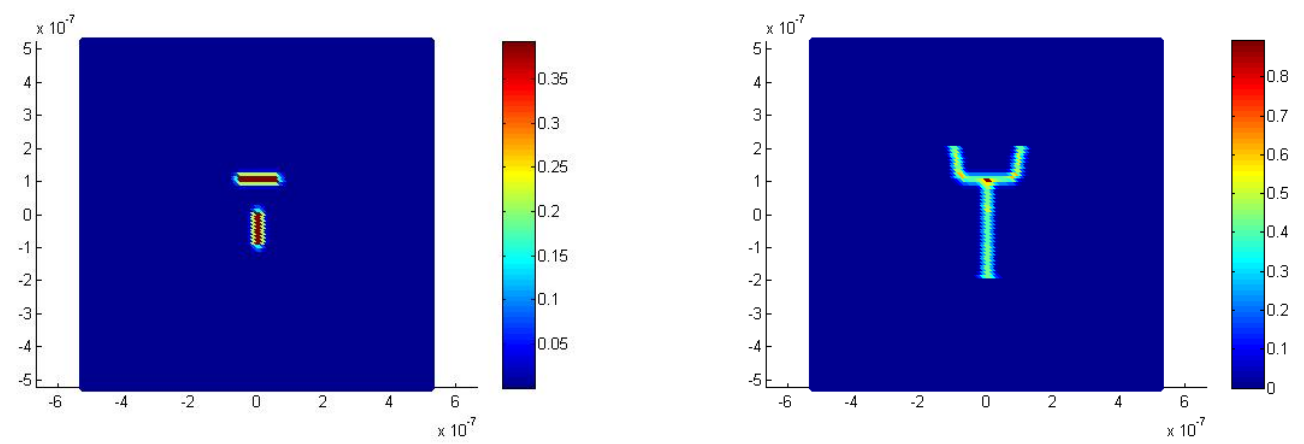

(a)

(b)
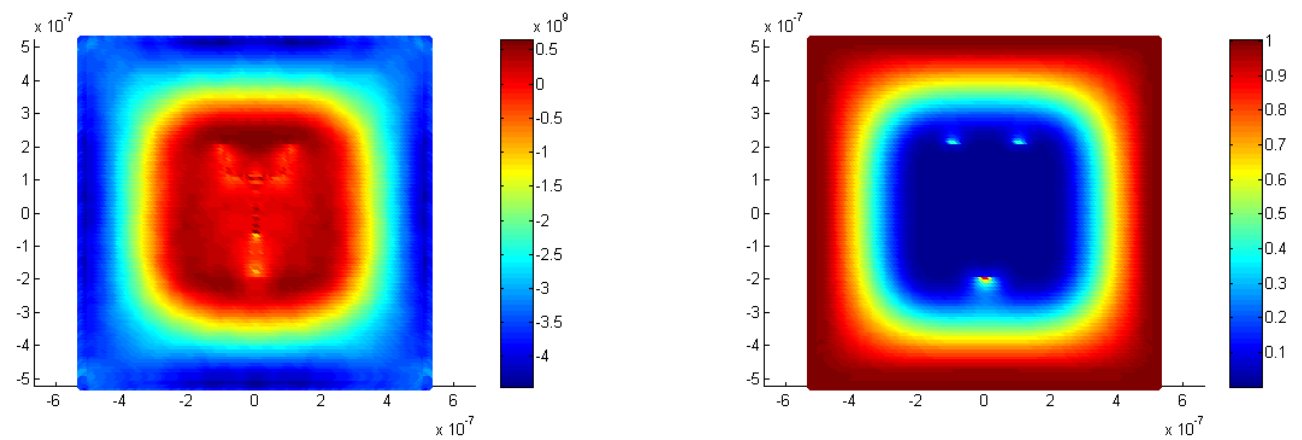

(c)

(d)

Figure 6: Results of electrode plate with perpendicular cracks: (a) Initial damage (b) Damage after deformation (c) Hydrostatic stress (d) Lithium ion concentration

In general conditions, cracks in battery electrode may have arbitrary arrangements. Therefore, two different cases; triple cracks damage situation and two oblique oriented cracks damage situation, are under investigation. In triple cracks analysis, cracks are located at the central plate and each crack length is $10 \%$ of the plate length. Oblique oriented cracks analysis has the same crack parameters as in the previous case shown in Fig. 6. However, the angle between the two cracks has changed from $90^{\circ}$ to $130^{\circ}$. The results of these two cases are given in Figs. 7 and Fig. 8.
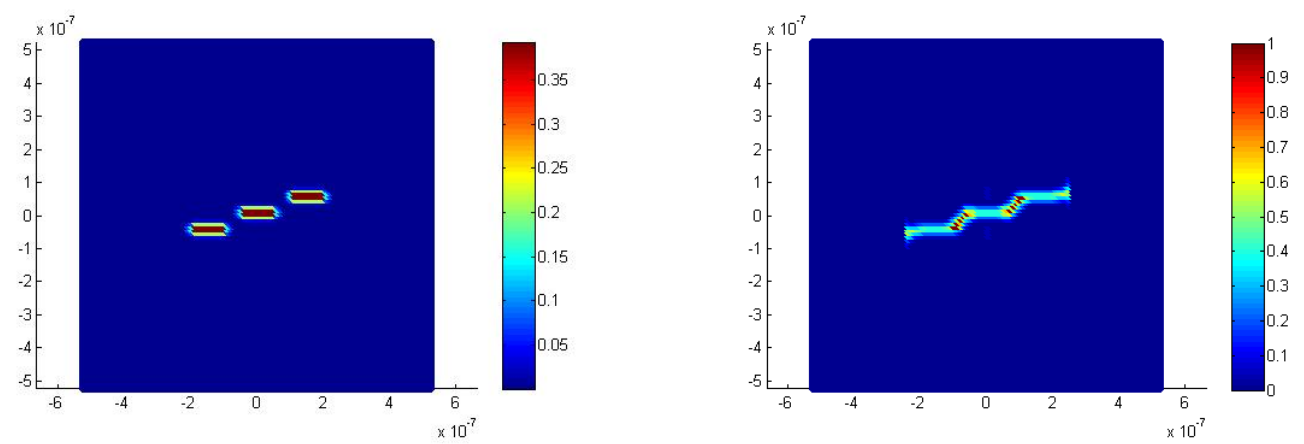

(a) 


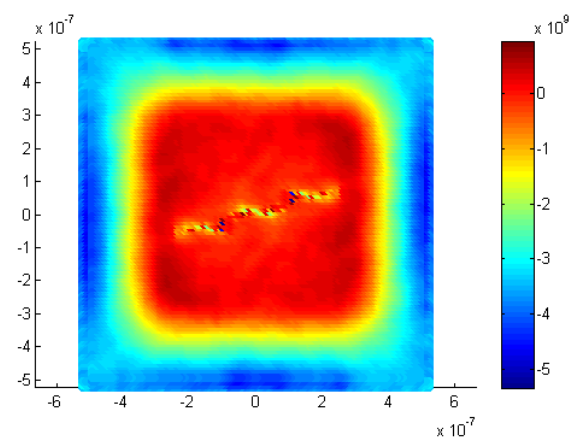

(c)

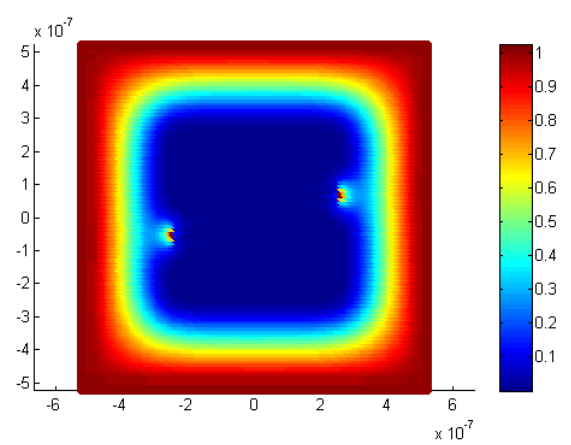

(d)

Figure 7: Results of the electrode plate with triple cracks: (a) Initial damage (b) Damage after deformation (c) Hydrostatic stress (d) Lithium ion concentration

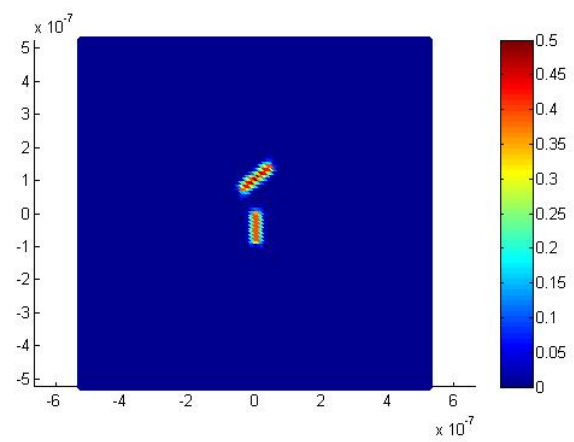

(a)

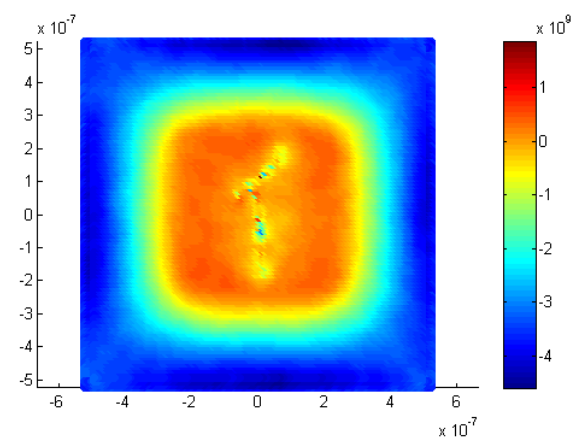

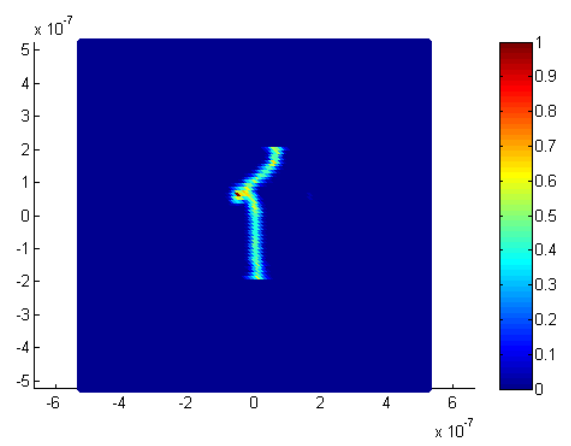

(b)

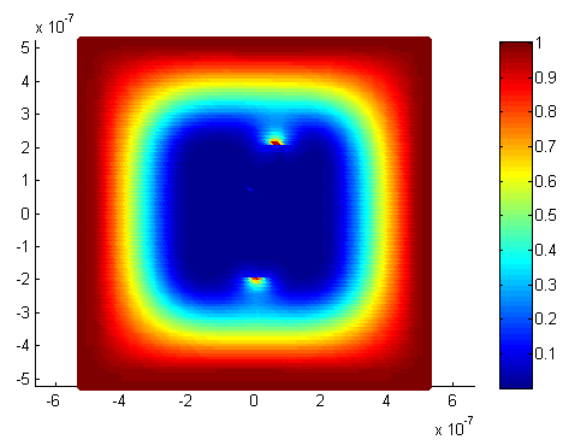

(c)

(d)

Figure 8: Results of the electrode plate with two oblique oriented cracks: (a) Initial damage (b) Damage after deformation (c) Hydrostatic stress (d) Lithium ion concentration

In triple crack analysis, since cracks are located close to each other, material points between the cracks have fewer interactions in their horizons and their stiffness are relatively weak. Therefore, central crack propagates towards to the neighbouring cracks from both crack tips and all three cracks merge into a single crack as shown in Fig. 7b. Propagation of the newly created crack proceeds horizontally through the electrode plate. According to Fig. 7c and Eq. 1, high hydrostatic stresses are concentrated at the outer crack tips and they are the main drivers of lithium ion 
enrichment. Hence, concentration of lithium ions at the outer crack tips is relatively higher than neighbouring points as shown in Fig. $7 \mathrm{~d}$.

For the two oblique oriented crack case, the two cracks merge into a " $\mathrm{T}$ " shape crack due to weak stiffness of material points between cracks. Since lithium ion concentration at the right crack tip is larger than the left crack tip, right crack tip propagates faster than the left crack tip as shown in Fig. 8b. In Fig. 8c, high stresses concentrate at the right and bottom crack tips. Therefore, lithium ion concentration at these crack tips is higher than surrounding particles.

Comparing the crack propagation patterns obtained in Sections 4.1 and 4.2 with the ones obtained in phase-field study [17], there is a very good match between the two studies which clearly shows that peridynamics can accurately predict crack propagation during the lithiation process.

\subsection{Coupled analysis for randomly located cracks}

After validating the developed methodology, its capabilities will be further demonstrated in this section. As a result of several cycling processes, lithium ion battery electrodes may accumulate large number of cracks. The shape and propagation direction of these cracks depend on the design of battery electrode plate [21].

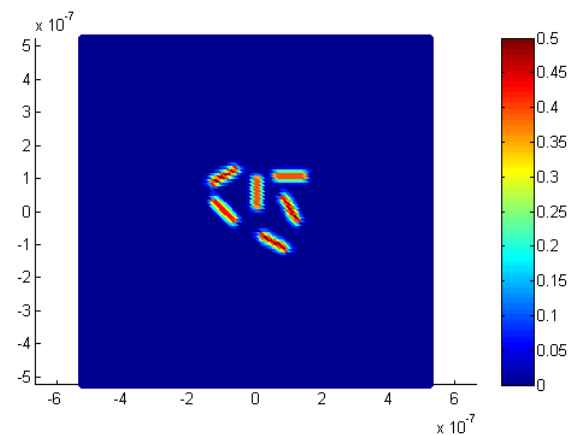

(a)

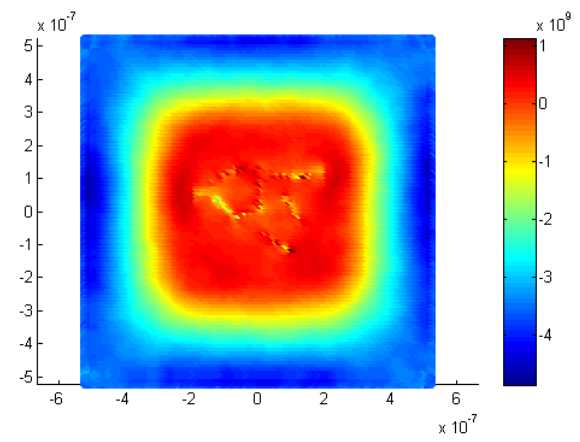

(c)

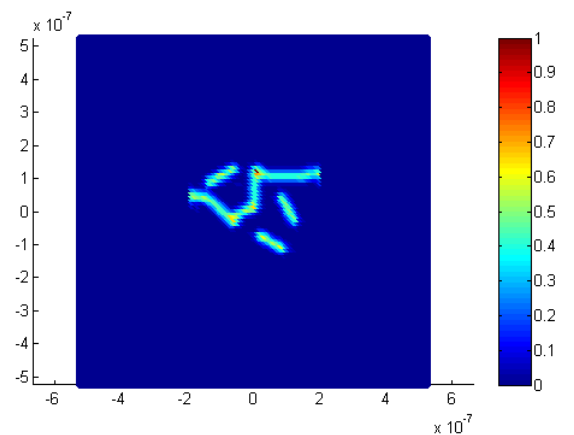

(b)

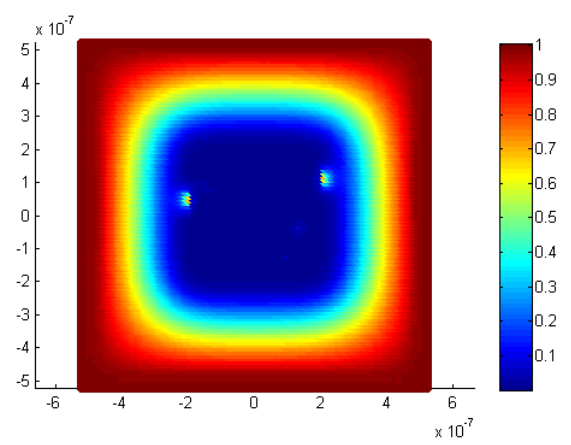

(d)

Figure 9: Results of the electrode plate with 6 randomly located cracks: (a) Initial damage (b) Damage after deformation (c) Hydrostatic stress (d) Lithium ion concentration 


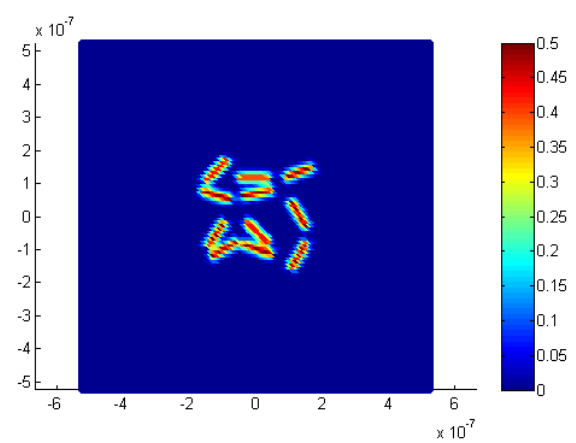

(a)

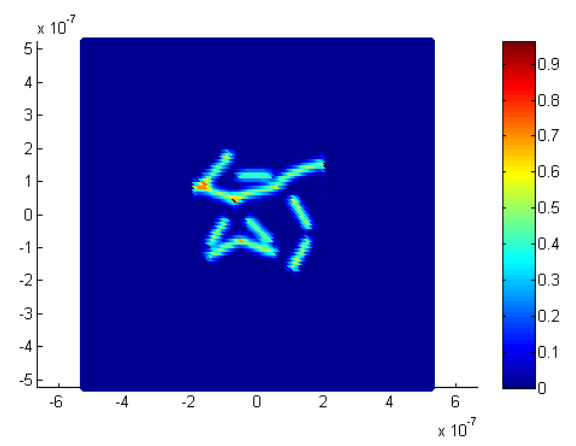

(b)

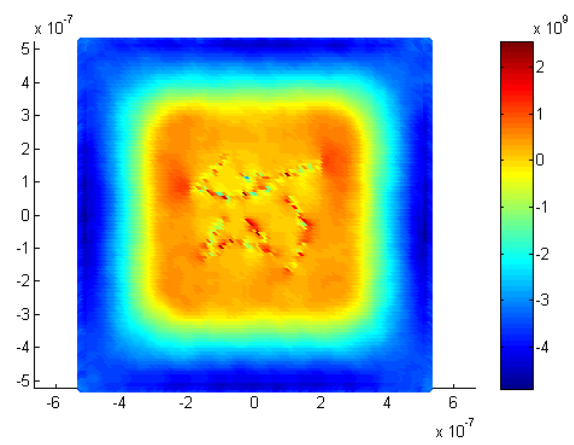

(c)

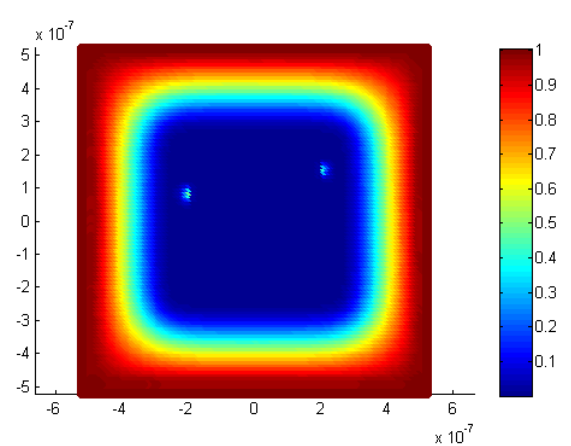

(d)

Figure 10: Results of the electrode plate with 11 randomly located cracks: (a) Initial damage (b) Damage after deformation (c) Hydrostatic stress (d) Lithium ion concentration

Two multiple crack battery plate specimens with 6 cracks and 11 cracks are chosen to represent two different damage situations after several battery cycling (Fig 9a and Fig. 10a). Each crack has a $10 \%$ of specimen length and is randomly located at the central plate. As lithium ions diffuse into the crack tip regions, hydrostatic stress increase dramatically at the crack tips. Hence, some inner cracks merge into a larger crack (Fig. $9 \mathrm{~b}$ and Fig. 10b) according to Eq. 1 and Eq. 11. However, other cracks especially those surrounded by the larger crack almost do not propagate. Once small cracks merge into a larger crack, hydrostatic stress at the crack tip regions reduces which may be the reason that the remaining small cracks stop propagating. In other words, the newly formed large crack doesn't allow the small cracks to propagate. On the other hand, outer crack tips of the larger crack are not affected by other cracks and propagate towards to the high concentration region. Hence, the concentration at outer crack tip region becomes higher than surrounding regions according to Eq. 1 (Fig. 9d and Fig.10d).

\section{Conclusions}

In this study, a new numerical simulation method, peridynamics, is utilized to analyse the fracture of lithium ion battery plates with pre-existing cracks. According to the 
numerical results, high hydrostatic stresses at the crack tips are the main motivation of crack propagation. As hydrostatic stresses increase at the crack tip region, lithium ion concentration grows as well and this leads to higher concentration values at the crack tip regions. Lithium ion battery electrode may have various damage situations and cracks normally propagate through material points with weak stiffnesses and high hydrostatic stresses. Hence, for multi-crack cases, some of the cracks merge into a larger crack first and then the large crack propagates from outer crack tips. Due to the merge of the crack, high hydrostatic stresses at some of crack tips reduce and this may arrest remaining cracks.

Based on the numerical results, it can be concluded that peridynamics provides a good estimation of damage evolution of lithium ion electrode plates. It can allow to predicting crack propagation without using remeshing. By using peridynamics, we can have a better understanding on failure mechanism in marine lithium ion batteries which can be used for designing better and more reliable batteries in the future. As a future study, the current formulation will be extended to a 3-Dimensional formulation and verified against experimental results available in the literature.

\section{References}

1. Herdzik, J., 2011. Emissions from marine engines versus IMO certification and requirements of tier 3. Journal of KONES, 18, pp.161-167.

2. Chen, W., Ådnanses, A.K., Hansen, J.F., Lindtjørn, J.O. and Tang, T., 2010, September. Super-capacitors based hybrid converter in marine electric propulsion system. In Electrical Machines (ICEM), 2010 XIX International Conference on (pp. 1-6). IEEE.

3. Dougherty, T.J., Segall, W.P. and Iverson, M.E., Johnson Controls Technology Company, 2002. Battery system. U.S. Patent 6,452,361.

4. Rosenkranz, C., 2003, November. Deep-cycle batteries for plug-in hybrid application. In EVS20 Plug-In Hybrid Vehicle Workshop, Long Beach, CA. Vancouver

5. Chung, S.Y., Bloking, J.T. and Chiang, Y.M., 2002. Electronically conductive phospho-olivines as lithium storage electrodes. Nature materials, 1(2), pp.123-128.

6. Zhou, Z., Benbouzid, M., Charpentier, J.F., Scuiller, F. and Tang, T., 2013. A review of energy storage technologies for marine current energy systems. Renewable and Sustainable Energy Reviews, 18, pp.390-400.

7. Daniel, C., 2008. Materials and processing for lithium-ion batteries. Jom, 60(9), pp.43-48. 
8. Grantab, R. and Shenoy, V.B., 2012. Pressure-gradient dependent diffusion and crack propagation in lithiated silicon nanowires. Journal of the Electrochemical Society, 159(5), pp.A584-A591.

9. Silling, S.A., 2000. Reformulation of elasticity theory for discontinuities and longrange forces. Journal of the Mechanics and Physics of Solids, 48(1), pp.175-209.

10. Madenci, E. and Oterkus, E., 2014. Peridynamic theory and its applications (Vol. 17). New York: Springer.

11. Oterkus, S., Madenci, E. and Agwai, A., 2014. Fully coupled peridynamic thermomechanics. Journal of the Mechanics and Physics of Solids, 64, pp.1-23.

12. Stamps, M.A. and Huang, H.Y.S., 2012, November. Mixed modes fracture and fatigue evaluation for lithium-ion batteries. In ASME 2012 International Mechanical Engineering Congress and Exposition (pp. 97-103). American Society of Mechanical Engineers.

13. Malvern, L.E., 1969. Introduction to the Mechanics of a Continuous Medium (No. Monograph).

14. Madenci, E., Barut, A. and Futch, M., 2016. Peridynamic differential operator and its applications. Computer Methods in Applied Mechanics and Engineering, 304, pp.408-451.

15. Chan, C.K., Peng, H., Liu, G., McIlwrath, K., Zhang, X.F., Huggins, R.A. and Cui, Y., 2008. High-performance lithium battery anodes using silicon nanowires. Nature nanotechnology, 3(1), pp.31-35.

16. Liu, X.H., Zhong, L., Huang, S., Mao, S.X., Zhu, T. and Huang, J.Y., 2012. Sizedependent fracture of silicon nanoparticles during lithiation. Acs Nano, 6(2), pp.15221531 .

17. Zuo, P. and Zhao, Y.P., 2015. A phase field model coupling lithium diffusion and stress evolution with crack propagation and application in lithium ion batteries. Physical Chemistry Chemical Physics, 17(1), pp.287-297.

18. Gao, Y.F. and Zhou, M., 2013. Coupled mechano-diffusional driving forces for fracture in electrode materials. Journal of Power Sources, 230, pp.176-193.

19. Vazic, B., Wang, H., Diyaroglu, C., Oterkus, S. and Oterkus, E., 2017. Dynamic propagation of a macrocrack interacting with parallel small cracks. AIMS Materials Science, 4(1), pp.118-136.

20. Ritchie, R.O., Yu, W. and Bucci, R.J., 1989. Fatigue crack propagation in ARALL ${ }^{\circledR}$ LAMINATES: measurement of the effect of crack-tip shielding from crack bridging. Engineering Fracture Mechanics, 32(3), pp.361-377. 
21. Li, J., Dozier, A.K., Li, Y., Yang, F. and Cheng, Y.T., 2011. Crack pattern formation in thin film lithium-ion battery electrodes. Journal of The Electrochemical Society, 158(6), pp.A689-A694.

22. Zimmermann, M., 2005. A continuum theory with long-range forces for solids (Doctoral dissertation, Massachusetts Institute of Technology).

23. Oterkus, S., 2015. Peridynamics for the solution of multiphysics problems. The University of Arizona.

24. Wang, H., Oterkus, E. and Oterkus, S., 2018. Predicting fracture evolution during lithiation process using peridynamics. Engineering Fracture Mechanics (under review).

25. Oterkus, S., Madenci, E. and Agwai, A., 2014. Peridynamic thermal diffusion. Journal of Computational Physics, 265, pp.71-96. 